\section{ALTO USO DE ANTICONCEPCIÓN ORAL DE EMERGENCIA EN PERÚ: RECONSIDERACIÓN DE SU LIBRE ACCESO}

\author{
INCREASED USE OF ORAL EMERGENCY \\ CONTRACEPTION IN PERU: RECONSIDERING \\ FREE ACCESS TO IT
}

Diana Quispe-Pineda',a, Kirbeliz Rebolledo-Ponietsky1,a, Antonelhla M. Ganoza-Calero ${ }^{1, a}$, José Miranda-Medina ${ }^{1, a}$, Natalia Elejalde-Farfán ${ }^{1, a}$, Diego Arévalo-Revelo ${ }^{1, a}$, Reneé Pereyra-Elías ${ }^{1, b}$

Sr. Editor. En octubre de 2009, el Tribunal Constitucional (TC) del Perú declaró inconstitucional la distribución gratuita del anticonceptivo oral de emergencia levonorgestrel (AOE), comúnmente conocido como "píldora del día siguiente". Esta decisión se basó en los principios pro homine y pro debilis ante el supuesto potencial abortivo del medicamento. En consecuencia, le prohibió al Ministerio de Salud desarrollar dicha distribución en los establecimientos del sector público ${ }^{(1)}$. Sin embargo, su venta en forma privada no fue vedada. No hemos encontrado publicaciones científicas acerca de la proporción de mujeres peruanas que hace uso del AOE.

Es por esto que deseamos comunicar los resultados de un estudio transversal ejecutado en mayo del 2016 en una muestra por conveniencia de mujeres entre 18 y 40 años asistentes a gimnasios de tres distritos de Lima (San Borja, Santiago de Surco, Miraflores). Evaluamos variables sociodemográficas, relaciones sexuales en los últimos seis meses (definición de "sexualmente activa" para este estudio) y el autorreporte de conocimiento del AOE (sí vs. no). Luego de una explicación escrita del AOE - que incluía el término "píldora del día siguiente"se preguntó por el uso del AOE alguna vez en la vida y al menos una vez en los últimos seis meses.

De 333 participantes, 299 contestaron las variables de interés. La mediana de edad fue 23 años [RIQ: 21-27], el $18,4 \%$ eran casadas/convivientes y el nivel mínimo de instrucción completado fue secundaria (34,5\%). El $88,0 \%$ había tenido relaciones sexuales con un hombre en los últimos seis meses. Aproximadamente, el 90,4\% de las participantes refirió conocer acerca del AOE. El

\footnotetext{
1 Escuela de medicina, Universidad Peruana de Ciencias Aplicadas. Lima, Perú. astudiante de Medicina; ${ }^{\mathrm{b}}$ médico cirujano

Recibido: 15/08/2016 Aprobado: 26/10/2016
}

Citar como: Quispe-Pineda D, Rebolledo-Ponietsky K, GanozaCalero AM, Miranda-Medina J, Elejalde-Farfán N, Arévalo-Revelo D, Pereyra-Elías R. Alto uso de anticoncepción oral de emergencia en Perú: reconsideración de su libre acceso. [carta]. Rev Peru Med Exp Salud Publica. 2016;33(4):839-40. doi: 10.17843/rpmesp.2016.334.2581
$67,6 \%$ manifestó haber usado el AOE por lo menos una vez en su vida y $45,8 \%$ lo había usado en los últimos seis meses. En la Figura 1, presentamos las frecuencias de uso del AOE para el total de mujeres y para las mujeres sexualmente activas.

Nuestros resultados son similares a los encontrados en la Encuesta Nacional de Demografía y Salud Familiar (ENDES) 2015. En este reporte, 21,2\% de todas las mujeres entre 15 y 49 años había usado el AOE por lo menos una vez. La prevalencia de vida de su uso entre

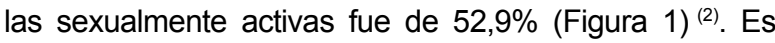
probable que las proporciones de uso en nuestra muestra sean mayores porque el denominador de la ENDES incluye a mujeres mayores a 40 años y menores de 18, que tienen menor probabilidad de haberla usado ${ }^{(2)}$. Por otro lado, por la naturaleza de la muestra de este estudio, las mujeres incluidas han sido probablemente de un estatus socioeconómico más alto. Esto supondría un mayor poder adquisitivo y, por ende, acceso al AOE.

Se ha descrito ampliamente que ciertos factores favorecen que los anticonceptivos modernos de uso rutinario no sean usados o no logren alcanzar su eficacia teórica. El conocimiento de estos métodos, el acceso económico y el contexto cultural en el que se desenvuelve una mujer se encuentran dentro de los principales ${ }^{(3)}$. El Perú es un escenario donde estos factores confluyen ${ }^{(2)}$. Es por esto que la Organización Mundial de la Salud califica a la anticoncepción de emergencia como esencial dentro de un sistema de salud ${ }^{(4)}$. De este modo, se previenen eficazmente embarazos no planificados y sus consecuencias (complicaciones obstétricas, abortos ilegalmente practicados, pobreza, entre otros) ${ }^{(3,5)}$.

Según lo expuesto, nuestra población se encuentra educada en sexualidad de manera subóptima y existen factores socioculturales que pueden determinar que no se utilicen anticonceptivos de rutina ${ }^{(2)}$. En tanto, el acceso a un método de emergencia es indudablemente necesario. El restringir su acceso solo para las mujeres que puedan comprarlo constituye una inequidad flagrante avalada políticamente ${ }^{(1)}$. Además, es una violación de los derechos reproductivos de las mujeres, ya que les imposibilita de decidir el número de hijos deseado ${ }^{(1,3)}$.

Acerca del "potencial abortivo" del medicamento, motivo fundamental de su prohibición, la evidencia generada a la actualidad es contundente. Revisiones de estudios sólidamente diseñados reportan que mecanismo de acción del AOE subyace, principalmente, en la inhibición o retraso en la ovulación ${ }^{(3)}$. No se ha demostrado que cause cambios importantes en el endometrio ni generación de condiciones antiimplantatorias o que prevengan el desarrollo del embrión luego de que se haya anidado satisfactoriamente ${ }^{(3,5)}$. Por lo tanto, la evidencia científica disponible hasta el momento 


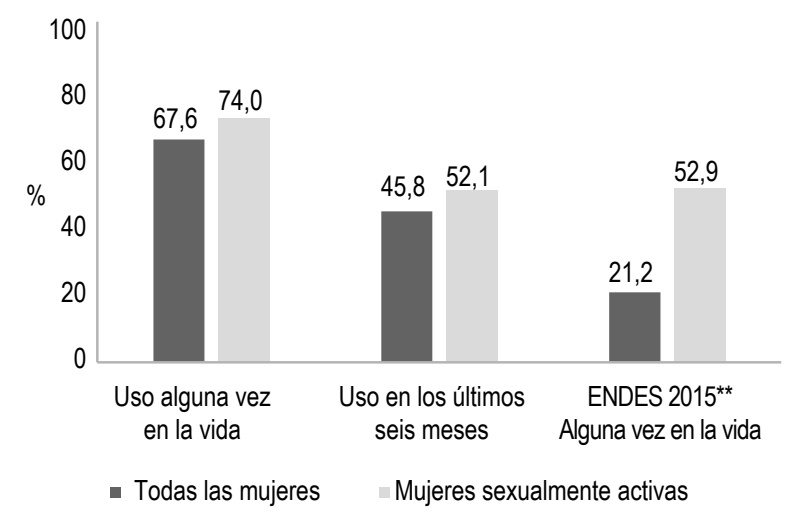

* Se consideró como sexualmente activa a quienes tuvieron relaciones sexuales con un varón en los últimos seis meses. La ENDES $2015^{(2)}$ consideró el último mes para esta definición.

** La ENDES 2015 evaluó a mujeres entre 15 y 49 años ${ }^{(2)}$.

Figura 1. Frecuencia de uso del anticonceptivo oral de emergencia en una muestra de mujeres de Lima 18 a 40 años $(n=299)$ y su comparación con información de la Encuesta Demográfica y de Salud Familiar - ENDES 2015

no indica que el AOE tenga efectos abortivos. Como consecuencia final, podemos afirmar que el fallo del TC no se sustenta en el conocimiento científico actual.

Concluimos que dos de cada tres mujeres de la muestra estudiada habían usado el AOE al menos una vez en su vida y casi la mitad lo había usado en los últimos seis meses. Si bien, la base de la planificación familiar es la educación y los métodos anticonceptivos de rutina, la necesidad del AOE en el sistema de salud peruano es ineludible. Se sugiere integrar nuevamente el tema en la agenda de salud pública y se reconsidere su distribución gratuita.

Contribución de autoría: DQP, KRP, AMGC, JMM, NEF y DAR participaron en la concepción del estudio, DQP, KRP,
AMGC, JMM, NEF, DAR y RPE participaron en el diseño del estudio, DQP, KRP, AMGC, JMM, NEF y DAR. participaron en la recolección de datos, RPE y DQP participaron en análisis de datos, DQP, KRP, AMGC, JMM, NEF, DAR y RPE participaron en la interpretación de información, DQP y AMGC participaron en redacción de versión preliminar, DQP, KRP, JMM, NEF, y RPE participaron en contribuciones intelectuales al manuscrito, DQP, KRP, AMGC, JMM, NEF, DAR y RPE aprobaron versión final, DQP, KRP, AMGC, JMM, NEF, DAR y RPE tienen responsabilidad por el contenido del artículo.

Conflictos de interés: uno de los autores es agnóstico; los demás, católicos.

Financiamiento: autofinanciado

\section{REFERENCIAS BIBLIOGRÁFICAS}

1. Pretell-Zárate EA. Política de anticoncepción oral de emergencia: la experiencia peruana. Rev Peru Med Exp Salud Publica. 2013;30(3):487-93.

2. Instituto Nacional de Estadística e Informática. Perú: Encuesta Demográfica y de Salud Familiar - ENDES 2015. Lima: INEI; 2015.

3. ESHRE CapriWorkshop Group. Emergency contraception. Widely available and effective but disappointing as a public health intervention: a review. Hum Reprod. 2015;30(4):751-60.

4. World Health Organization. 19th WHO Model List of Essential Medicines (April 2015). Geveva: WHO; 2015.

5. Raymond EG, Cleland K. Clinical practice. Emergency contraception. N Engl J Med. 2015;372(14):1342-8.

Correspondencia: Reneé Pereyra-Elías

Correo electrónico: renee.pereyra.elias@gmail.com

Dirección: Av. Alameda San Marcos, Cda 2, Chorrillos, Lima 09.

Lima, Perú. 